\title{
A non-perturbative Kolmogorov turbulence approach to the cosmic web structure
}

\author{
JosÉ GaITE
}

\author{
PACS 98.65.Dx - Superclusters; large-scale structure of the Universe (including voids, pancakes, \\ great wall, etc.) \\ PACS 47.27.-i - Turbulent flows \\ PACS 05.45.Df - Fractals
}

\begin{abstract}
The Kolmogorov approach to turbulence is applied to the Burgers turbulence in the stochastic adhesion model of large-scale structure formation. As the perturbative approach to this model is unreliable, here a new, non-perturbative approach, based on a suitable formulation of Kolmogorov's scaling laws, is proposed. This approach suggests that the power-law exponent of the matter density two-point correlation function is in the range 1-1.33, but it also suggests that the adhesion model neglects important aspects of the gravitational dynamics.
\end{abstract}

The large-scale structure of the universe is produced by the gravitational clustering of an initially homogeneous matter distribution. This process can be described by the Newtonian equations of motion of the matter fluid, written in comoving coordinates and in terms of the peculiar velocity and gravitational fields [1]. These equations are nonlinear and, although they can be linearized to describe the early growth of small perturbations of the initially homogeneous distribution, the actual structure formation takes place when the nonlinearity plays a major role, at the aptly called nonlinear stage of gravitational clustering. The nonlinearity of fluid mechanics also plays a major role in the phenomenon of turbulence and this is the cause of the difficulty in treating this phenomenon (often referred to as the "unsolved problem of classical physics"). Since turbulence is a key actor in many astrophysical scenarios, it is tempting to apply methods and ideas of turbulence to the study of large-scale structure formation.

An early attempt to apply Kolmogorov's scaling laws to the origin of galaxies was made by von Weizsäcker [2], but his ideas have been since mostly restricted to intragalactic turbulence and have played no role in the study of the formation of clusters and superclusters of galaxies or the large-scale distribution of dark matter. However, a popular model of large-scale structure formation, namely, the adhesion model [3-5], is essentially a model of pressureless turbulence, that is to say, the type of turbulence that occurs in strongly compressible potential flows and is usually called Burgers turbulence. As shown in this letter, the Kolmogorov approach to turbulence can be applied to cosmic Burgers turbulence, employing a suitable formulation of the adhesion model.

In cosmology, scaling laws for the velocity field, such as Kolmogorov's laws, are especially important if they can be related to scaling laws for the matter density field, because the positions of astronomical objects are more easily measurable than their velocities and indeed have been shown to obey scaling laws. The best known scaling laws in cosmology have been found in the distribution of galaxies [6,7]. The first and prototypical scaling law is the power law of the galaxy-galaxy correlation function, which is proportional to $r^{-\gamma}$, where $r$ is the inter-galaxy distance. This law suggests a fractal distribution of galaxies, but more detailed analyses show that a more general, multifractal distribution is more appropriate [6,7]. Even more detailed analyses, namely, analyses of the full matter distribution, including the dark matter, are possible with the help of $N$-body cosmological simulations, and they show that both the dark matter and the baryonic matter form a common multifractal "cosmic web" structure [8]. The cosmic web is precisely the type of structure predicted by the adhesion model [3-5].

The cosmic web consists of sheets (Zeldovich "pancakes"), filaments and nodes, so it is indeed 
multifractal, in the sense that it is formed by objects of several dimensions, namely, two, one and zero dimensions. However, such a distribution needs not be scale invariant. Nevertheless, the actual cosmic web structure is, arguably, a self-similar multifractal [8]. The reason why the adhesion model does not necessarily predict a scale-invariant distribution lies in a peculiarity of the Burgers equation, which we now explain. This equation writes

$$
\frac{\partial \boldsymbol{u}}{\partial t}+\boldsymbol{u} \cdot \nabla \boldsymbol{u}=\nu \nabla^{2} \boldsymbol{u}
$$

where $u$ is the velocity and $\nu$ the viscosity coefficient ${ }^{1}$. To this equation, it must be adjoined the potential-flow condition, $\nabla \times \boldsymbol{u}=0$. Although the Burgers equation is a particular case of the general Navier-Stokes equation, it has the peculiarity of being integrable, in sharp contrast with the more usual Navier-Stokes equation of incompressible turbulence. In other words, the chaotic properties of the latter are not present in the former, which nicely evolves the initial conditions. Therefore, the natural way of producing a self-similar cosmic web structure is by using self-similar initial conditions, namely, an initial Gaussian velocity distribution with a power-law power spectrum [5]. A distribution of this type evolves to a self-similar cosmic web.

The exact integral of the Burgers equation is obtained through the Hopf-Cole transformation, which transforms eq. (1) into the standard heat equation [3-5]. In the zeroviscosity limit, $\nu \rightarrow 0$, the exact integral gives rise to a simple geometrical construction, in terms of Lagrangian coordinates (common in fluid mechanics). Equation (1) for $\nu=0$ is just $\mathrm{d} \boldsymbol{u} / \mathrm{d} t=0$, so fluid particles actually move along different straight lines, until certain groups of nearby particles collide at caustic surfaces. The straight lines can be prolonged beyond the caustics, defining multistreaming regions, where the field $\boldsymbol{u}$ given by $\mathrm{d} \boldsymbol{u} / \mathrm{d} t=0$ is multivalued. However, in caustics, the viscous term of eq. (1), $\nu \nabla^{2} u$, is non-vanishing even if $\nu \rightarrow 0$, because $\nabla^{2} u$ then diverges. The analysis of the Hopf-Cole solution shows that any infinitesimal viscosity prevents multistreaming and just produces the adhesion of particles at caustics, which become shock fronts (u-discontinuities). When the initial velocity distribution is Gaussian with a power-law power spectrum, so that the initial velocity field is continuous but non-smooth, shock fronts are formed after an arbitrarily short time and have a built-in selfsimilarity [5]. This shock-front structure constitutes a self-similar cosmic web.

For $\nu=0$, eq. (1) is indeed scale invariant, in the sense that it is invariant under simultaneous space and time scalings $\lambda \boldsymbol{x}, \lambda^{1-h} t$, for arbitrary $h$, if the velocity is scaled as $\lambda^{h} u$. Therefore, if the initial velocity field is scale

\footnotetext{
${ }^{1}$ As regards the cosmological application of eq. (1), it must be noticed that $t$ is not the standard time but a function of it, namely, the growth rate of linear density fluctuations [3-5].
}

invariant with exponent $h$, the solution of the inviscid equation obeys the dynamical scaling law

$$
\boldsymbol{u}(\boldsymbol{x}, t)=t^{h /(1-h)} \boldsymbol{u}\left(\boldsymbol{x} / t^{1 /(1-h)}, 1\right)
$$

that is, the solution at any $t>0$ is obtained by scaling the solution at $t=1$. Naturally, this scaling law can be derived from the $\nu \rightarrow 0$ limit of the Hopf-Cole integral solution for an initial velocity distribution that is Gaussian with a power-law power spectrum, in which case, the natural range of the exponent $h$ is $-1<h<1[5]$. The dynamical scaling law (2) is connected with a dynamical invariant, $u^{1 / h} / x$, which can be identified with the specific dissipation rate $\varepsilon$ for $h=1 / 3$ (the Kolmogorov scaling). One can further deduce that there is a homogeneity scale $L(t)=t^{1 /(1-h)} L(1)$, such that the cosmic web structure at time $t$ has only formed on scales smaller than $L(t)$ whereas the initial homogeneous distribution stays on larger scales. Indeed, the initial conditions are recovered by eq. (2) in the $t \rightarrow 0$ limit, which is singular but is essentially attained if $x \gg L(t)$. The homogeneity scale $L$ plays a role that is similar to the role of the integral scale in incompressible Navier-Stokes turbulence. On the other hand, $L$ can be identified with a standard scale in cosmology, namely, $r_{0}$, which denotes the scale at which the galaxy-galaxy correlation function is equal to one (and is sometimes given the misnomer "correlation length") [9].

Equation (1) only constrains the velocity field, and we would like to relate it to the matter density field. This field is given by the motion of fluid particles, but it cannot be directly obtained from the Hopf-Cole solution for $u$. Nevertheless, in the zero viscosity limit, a convenient expression of the density field in terms of the velocity field can be obtained $[10]$; namely

$$
\varrho(\boldsymbol{x})=\varrho_{0} \operatorname{det}\left[\delta_{i j}-\partial_{i} u_{j}(\boldsymbol{x})\right],
$$

where $\varrho_{0}$ is the constant initial density, and it is assumed that $t=1$. The determinant is actually the Jacobian determinant of the transformation to Lagrangian coordinates. Expression (3) simplifies to

$$
\delta \varrho(\boldsymbol{x}):=\varrho(\boldsymbol{x})-\varrho_{0}=-\varrho_{0} \partial_{i} u^{i}(\boldsymbol{x})
$$

in the linear regime, such that $|\delta \varrho| \ll \varrho_{0}$. In the nonlinear regime, caustics arise when the Jacobian determinant blows up and, according to eq. (3), $\varrho \rightarrow \infty$.

Unfortunately, the above-explained approach, based on the simple (integrable) evolution of an initial velocity distribution with suitable statistical properties, has obvious shortcomings: i) in cosmology, the initial power spectrum is not a power law; ii) the adhesion model is just a simplified model of the gravitational dynamics, which actually is chaotic; and iii) the adhesion of matter in shock fronts is considered as an inelastic collision but the dissipated energy is not accounted for. In general, chaos and dissipation are connected: chaotic dynamics erases memory of initial conditions, giving rise to an irreversible process in which entropy grows. Therefore, it is reasonable 
to supplement the adhesion model with a "noise", which reverts the lost kinetic energy, on the one hand, and makes the long-time evolution of the velocities independent of the initial conditions, on the other hand. The noise, or random force, is just added to the right-hand side of eq. (1).

This stochastic adhesion model possesses an attractor characterized by a dynamical scaling which, unlike the scaling defined by eq. (2), is independent of the initial conditions; namely, t-scaling occurs only if $t$ is interpreted as the time difference between two arbitrary events in the asymptotic stationary state. Nevertheless, this stationary state corresponds again to a cosmic web structure, which can be described as a "quasi-Voronoi" tessellation of shock fronts [11]. Moreover, the stationary state, in which the energy injected on scales $>L$ is dissipated at constant rate $\varepsilon$ at the Kolmogorov scale, is analogous to the stationary state of incompressible turbulence. However, in Burgers turbulence, the dissipation takes place in shock fronts and has more spatial variation than in incompressible turbulence, producing strong intermittency. Intermittency causes deviations from Kolmogorov's scaling in higherorder correlation functions, and these deviations must be taken into account, as shown below. $A$ beautiful exposition of Kolmogorov's ideas and of intermittency is given by Frisch [12].

The stochastic Burgers equation is well studied, since it is equivalent to the Kardar-Parisi-Zhang (KPZ) equation, which plays an important role in the physics of interface growth. The equivalence is realized by expressing the Burgers equation in terms of the velocity potential and identifying this potential with the interface's height. The KPZ equation includes a Gaussian noise with power spectrum $D(k, \omega)$. It has been studied with renormalized perturbation theory by Medina et al. [13]. With this method, the types of noise that give rise to dynamical scaling are determined as fixed points of the dynamical renormalization group. For white noise, in three spatial dimensions, the non-trivial fixed point is repulsive, so the nonlinear term of the Burgers equation is irrelevant (in the renormalization group sense) and the viscous term dominates in the perturbative stationary state. Therefore, turbulence can only occur in the strong-coupling, nonperturbative regime.

More generally, one can consider "colored" noise, but with just spatial correlations, namely, with spectrum $D(k)$, in order not to break Galilean invariance [13]. For small $k$, namely, length scales much larger than the Kolmogorov scale, one can assume the universal form $D(k) \approx D_{0}+D k^{-2 \rho}, \rho>0$, that is to say, the noise consists of white noise plus a power-law correlated noise. Unfortunately, the addition of power-law correlated noise does not bring real improvements: there can be several fixed points, but, in three dimensions, only the trivial fixed point is stable and only if $\rho$ is small; otherwise, it becomes a saddle point [13]. This means that a noise with sufficient power on large scales inevitably leads to a strong-coupling stationary state.
The method of Medina et al. [13] has been adapted to the cosmology setting by Domínguez et al. [14]. They consider a more general type of noise, which includes temporal correlations, namely, $D(k, \omega)=D_{0}+D k^{-2 \rho} \omega^{-2 \theta}$. Furthermore, their KPZ equation has an extra term, proportional to the velocity potential, that is, a term with a dimensional coupling that they call "mass", in analogy with quantum field theory. The corresponding renormalization group equations have several fixed points, but only one is stable. A choice of $\rho$ and $\theta$ in certain ranges yields a range of exponents of the power-law velocitypotential correlation function such that the corresponding range of exponents of the density correlation function, obtained through eq. (4), agrees with the observational range of $\gamma$ (the exponent of the galaxy-galaxy correlation function). This intriguing derivation of $\gamma$ has several questionable aspects, besides the ad-hoc choice of $\rho$ and $\theta$. First, Galilean invariance is broken, as the existence of the stable fixed point demands a non-zero $\theta$ [14]. Second, eq. (4) is only valid in the linear regime, in principle. Third, the results, apart from the value of $\gamma$, are also questionable: Regarding the values of the couplings at the fixed-point, one notices: the strength $D$ of the colored noise turns out to be negative (note that the colored noise dominates over the white noise for small $k$ ); furthermore, the "mass" scale is non-vanishing, so the stable fixed point does not seem to correspond to a scale-invariant stationary state.

At any rate, one can argue, on general grounds, that perturbation theory (especially, the one-loop approximation) is not the right approach to Burgers turbulence. The effective coupling constants in the renormalization group equations have the generic expression $\lambda^{2} D / \nu^{3}$ (except the "mass"), where $\lambda$ is the nonlinear coupling constant (to be set to the value of unity), $D$ is a noise strength, and $\nu$ the viscosity. Therefore, as the nonlinear term dominates in the inertial range, the coupling must be strong. More precisely, the given expression implies that the coupling constants are actually proportional to the cube of the Reynolds number, which has to be a very large number, making perturbation theory unreliable.

Therefore, one must resort to non-perturbative methods. Standard non-perturbative methods of turbulence are the closure approaches, in which the hydrodynamical hierarchy of equations for statistical moments is closed at some order by assuming a relation between the moments of the corresponding order and lower-order ones. There is a similar closure approach in cosmology, based on the BBGKY hierarchy [1]. This a second-order closure and it is consistent with a scaling ansatz for the two-point correlation functions, with just one power-law exponent, but this number remains undetermined, unless it is connected with the initial conditions, namely, with an initial power-law power spectrum of perturbations. Since we avoid this type of connections, on account of the chaotic nature of gravitational dynamics, we prefer to follow the traditional non-perturbative methods of turbulence. They 
are based on reasonable assumptions, the simplest ones being Kolmogorov's universality assumptions, namely, homogeneity, isotropy, and scaling laws for the moments of longitudinal velocity increments [12]. These laws state that

$$
\left\langle(\delta \boldsymbol{u} \cdot \boldsymbol{r} / r)^{n}\right\rangle \propto(\varepsilon r)^{n / 3},
$$

where $\delta \boldsymbol{u}=\boldsymbol{u}(\boldsymbol{x}+\boldsymbol{r} / 2)-\boldsymbol{u}(\boldsymbol{x}-\boldsymbol{r} / 2)$ and $n \in \mathbb{N}$. A general form of the Kolmogorov scaling laws, suitable for introducing the effect of intermittency, is

$$
\left\langle|\delta \boldsymbol{u}|^{q}\right\rangle=A r^{\zeta(q)}
$$

where $q \in \mathbb{R}$, and $A$ does not depend on $r$. This general scaling law characterizes $\boldsymbol{u}$ as a (random) multifractal or multi-affine function, whereas the restriction to $\zeta(q) \propto q$ corresponds to an ordinary self-affine function [15]. Therefore, eq. (5) corresponds to a particular form of selfaffinity, whereas the effect of intermittency is to give rise to multifractality, given by the function $\zeta(q)$, whose properties are explained in the following.

Kolmogorov's scaling laws can be justified by employing the hierarchy of hydrodynamical equations, in particular, the second-order one, called the Karman-Howarth-Monin equation [12]. A version of this equation is valid for Burgers turbulence. An illuminating derivation of the equation has been given by Polyakov [16] in the onedimensional case. Polyakov realizes that, in the equation for $\partial_{t} u^{2}$, the dissipation in the inertial range arises as a field-theory anomaly, due to the non-differentiability of the velocity field. The form of the anomaly can be found by employing a point-splitting method (already implicit in the definition of $\delta \boldsymbol{u}$ ). In the three-dimensional case, the calculation is more involved but it yields the simple result

$$
\partial_{t} u^{2}(\boldsymbol{x})=-u^{i}(\boldsymbol{x}) \frac{\partial u^{2}(\boldsymbol{x})}{\partial x^{i}}+\frac{1}{2} \lim _{\boldsymbol{r} \rightarrow \mathbf{0}} \frac{\partial}{\partial r^{i}}\left(\delta u^{i} \delta \boldsymbol{u}^{2}\right)
$$

(in the $\nu \rightarrow 0$ limit). The last term is the anomaly $a(\boldsymbol{x})$, which would vanish if $u(x)$ were differentiable. Remarkably, to derive eq. (7), we do not need homogeneity or isotropy. Anyway, homogeneity and isotropy are part of Kolmogorov's universality assumptions and are natural in cosmology. From eq. (7), one deduces that the average dissipation in the steady state is $\varepsilon=-\langle a\rangle / 2$. This closure relation is an exact formulation of the $n=3$ case of the scaling laws (5) for Burgers turbulence, analogous to Kolmogorov's " $4 / 5$ " law of incompressible turbulence [12]. Therefore, in eq. (6), $\zeta(3)=1$ (assuming that $\varepsilon$ is well defined in the $\nu \rightarrow 0$ limit).

If the probability $P(\delta \boldsymbol{u})$ were Gaussian, then $\zeta(q) \propto q$ (self-affine $u$ ), and necessarily $\zeta(q)=q / 3$, as in eq. (5). However, intermittency manifests itself in a slower growth of $\zeta(q)$ for $q>3$, as extensively studied by Frisch [12] for incompressible turbulence. As already said, intermittency in Burgers turbulence is especially strong. Indeed, in one dimension and with power-law correlated noise, the strength of intermittency depends on the noise exponent but is always such that the maximum of $\zeta(q)$ is $\zeta=1$, as shown by Hayot and Jayaprakash (who qualify this behaviour as "extreme multifractality") ([17], especially, fig. 2). The reason for that maximum is that the average in eq. (6) is dominated, starting from some value of $q$, by the effect of shocks; so one gets, by replacing the ensemble average with a spatial average,

$$
\left\langle|\delta u|^{q}\right\rangle \sim \sum_{n}\left|\delta u_{n}\right|^{q} r
$$

where the sum is over the set of $u$-discontinuities and $\delta u_{n}$ is the jump at the $n$-th discontinuity.

Hayot and Jayaprakash [17] determine the values of $q$ at which $\zeta(q)$ reaches the value of unity, in terms of the exponent of the noise power-law correlation function. Their results can be generalized to three dimensions. In terms of the KPZ noise exponent $\rho$, the value $\rho=5 / 2$ is such that the noise strength $D$ has the dimensions of $\varepsilon$ and the (Burgers) noise correlation function is proportional to $\log r$. This leads to the Kolmogorov scaling law $\zeta(q)=q / 3$ for $q \leqslant 3$, while $\zeta(q)=1$ for $q \geqslant 3[17]$. The $r \rightarrow 0$ limit of the noise correlation function, equal to $\varepsilon$, diverges as $\nu \rightarrow 0$ for $\rho<5 / 2$ and diverges as $L \rightarrow \infty$ for $\rho>5 / 2$. In other words,

$$
\varepsilon=\int_{0}^{\infty} k^{2} D(k) \mathrm{d}^{3} k
$$

diverges at $k=\infty$ or at $k=0$ and therefore is not universal. However, the $r$-dependent part of the noise correlation function is universal and proportional to $r^{2 \rho-5}$ for $3 / 2<$ $\rho<7 / 2, \rho \neq 5 / 2$. In particular, the values of $\rho \in(5 / 2,7 / 2)$ correspond to large-scale forcing, such that $\varepsilon$ must depend on $L$ but is well defined in the $\nu \rightarrow 0$ limit. This large-scale forcing leads to $\zeta(q)=1$ if $q \geqslant 3$, like for $\rho=5 / 2$, but now $2 / 3<\zeta(2)<1$. For $\rho>7 / 2$, the $r$-dependent part of the noise correlation function is not universal and depends on what happens on scales of the order of $L$; that is to say, it is determined by the initial conditions. Furthermore, $\zeta(q)=1$ for $q \geqslant 2$, so $\zeta(2)$ reaches its maximum value. In this case, namely, $\rho>7 / 2$, the stochastic adhesion model is presumably equivalent to the ordinary adhesion model with self-similar initial conditions. Therefore, the interesting range for the cosmic structure is $\rho \in(5 / 2,7 / 2)$.

Our next step is to calculate the density correlation function from eq. (3), assuming eq. (6) with $\zeta(q)=1$ for $q \geqslant 3$ and $2 / 3<\zeta(2)<1$. The expansion of the determinant in eq. (3) yields

$$
\begin{aligned}
& \delta \varrho / \varrho_{0}=-\partial_{i} u^{i}+\left(\partial_{1} u^{1} \partial_{2} u^{2}-\partial_{1} u^{2} \partial_{2} u^{1}+\partial_{1} u^{1} \partial_{3} u^{3}\right. \\
& \left.-\partial_{1} u^{3} \partial_{3} u^{1}+\partial_{2} u^{2} \partial_{3} u^{3}-\partial_{2} u^{3} \partial_{3} u^{2}\right)+O(\partial u)^{3} .
\end{aligned}
$$

While eq. (4), of $O(\partial u)$, is valid in the linear regime, we have to consider the formation of caustics. As already said regarding eq. (3), caustics and hence density singularities are due to the blowing up of the Jacobian determinant, namely, of some eigenvalue(s) of the matrix $\partial_{i} u_{j}=\partial_{i j} \phi$, where $\phi$ is the velocity potential. When there are density 
singularities, the relative importance of the summands in the right-hand side of eq. (8) is given by the number of diverging eigenvalues. Therefore, eq. (4) may still hold in the nonlinear regime if only one eigenvalue diverges, that is to say, for the formation of sheets in the early stages, whereas for filaments or nodes, the remaining terms in eq. (8) are necessary. Let us see how this idea is realized for the density correlation function.

The reduced two-point correlation function of the density is

$$
\begin{aligned}
\langle\delta \varrho(\boldsymbol{r}) \delta \varrho(\mathbf{0})\rangle / \varrho_{0}^{2}= & \left\langle\partial_{i} u^{i}(\boldsymbol{r}) \partial_{j} u^{j}(\mathbf{0})\right\rangle-2 c(r) \\
& +\left\langle\mathrm{O}(\partial u)^{4}\right\rangle+\cdots,
\end{aligned}
$$

where

$$
\begin{aligned}
c(r)= & \left\langle\left(\partial_{1} u^{1} \partial_{2} u^{2}-\partial_{1} u^{2} \partial_{2} u^{1}+\partial_{1} u^{1} \partial_{3} u^{3}-\partial_{1} u^{3} \partial_{3} u^{1}\right.\right. \\
& \left.\left.+\partial_{2} u^{2} \partial_{3} u^{3}-\partial_{2} u^{3} \partial_{3} u^{2}\right)(\boldsymbol{r}) \partial_{j} u^{j}(\mathbf{0})\right\rangle .
\end{aligned}
$$

We have shown explicitly only terms up to $\mathrm{O}(\partial u)^{3}$, because the other terms do not require any calculation, as we now explain. The functions on the right-hand side of eq. (9) are power-laws of $r$, each one with a characteristic exponent $-\gamma$ that can be deduced from eq. (6); namely, $-\gamma=\zeta(n)-n$ for $\left\langle\mathrm{O}(\partial u)^{n}\right\rangle$. Given that $\zeta(n)=1$ for $n=$ $3,4,5,6$, we have $\gamma=2,3,4,5$, respectively. However, the maximal value is $\gamma=3$, which is the value for a Poisson distribution (shot-noise) term: this term can appear as either $\delta(\boldsymbol{r})$ or $r^{-3}$ (see, e.g., ref. [9]). In general, the value $\gamma=3$ appears whenever there are points with finite mass, as, for instance, the cosmic-web nodes. Since $\gamma=3$ is reached for $n \geqslant 4$, we only need to consider the cases $n=3$ and $n=2$.

To explicitly calculate $c(r) \propto r^{-2}$, it is useful to express it as $c(r)=\Delta g(r)$, where

$$
\begin{aligned}
g(r)= & \left\langle\left(\partial_{11} \phi \partial_{22} \phi-\partial_{12} \phi \partial_{21} \phi+\partial_{11} \phi \partial_{33} \phi-\partial_{13} \phi \partial_{31} \phi\right.\right. \\
& \left.\left.+\partial_{22} \phi \partial_{33} \phi-\partial_{23} \phi \partial_{32} \phi\right)(\boldsymbol{r}) \phi(\mathbf{0})\right\rangle .
\end{aligned}
$$

This function is a dimensionless scalar, so it must be a constant. Therefore, $c(r)=0$ and the $\gamma=2$ contribution vanishes.

When the density correlation function is, in the nonlinear domain $r \ll L$, the sum of different powers of $r$, the most singular term dominates on the smaller scales. The most singular term is, of course, the $r^{-3}$ term, but it must be discarded [9]. Therefore, in the end, the relevant contribution to the density two-point correlation function is just due to the velocity two-point correlation function, as if the only contribution to the density is due to sheets. The corresponding exponent is $\gamma=2-\zeta(2)$. Since $2 / 3<\zeta(2)<1$, we obtain $1<\gamma<4 / 3$. The Kolmogorov scaling $\zeta(2)=2 / 3$ yields the upper bound, $\gamma=4 / 3 \simeq 1.33$. The range of values of $\gamma$ obtained from galaxy surveys or $N$-body cosmological simulations is (mostly) in the interval $(1,2)[6-8]$. However, the classic value $\gamma=1.7$, which still stands $[7,8]$, is larger than $4 / 3$. Nevertheless, a specific methodology for the analysis of galaxy catalogs [6] yields values of $\gamma$ in the interval $(1,1.3)$.

To obtain values of $\gamma$ in the interval $(4 / 3,2)$, one could try a $\rho \in(3 / 2,5 / 2)$. Then, the Kolmogorov scale could not be set to zero, so it should be kept and, preferably, identified with a physical scale. In the gravitational dynamics, there is no intrinsic small scale, although there are small scales in the initial conditions. In $N$-body cosmological simulations, the most suitable small scale is the scale of gravitational smoothing. In any case, if we were to take $\rho \in(3 / 2,5 / 2)$, then $\zeta(3)<1$, so $c(r)$ would not vanish. As this term is more singular than $r \zeta(2)-2$, it would produce a $\gamma>2$ on the smaller scales, so the density correlation function would not be a pure power law with $\gamma \in(4 / 3,2)$ over the entire inertial range. This alteration of the smaller scales for $\rho \in(3 / 2,5 / 2)$ is related to the non-universality of $\varepsilon$ and, hence, of the Kolmogorov energy cascade, which both would depend on the detailed dissipation mechanism below the Kolmogorov scale.

With $1 \leqslant \gamma<4 / 3$, the density correlation function is dominated by sheets. Interestingly, the analysis of $N$-body cosmological simulations leads one to a similar conclusion: the bulk of mass belongs to sheets [8]. However, to fully understand the role of the three types of cosmic-web singularities, namely, sheets, filaments and nodes, one must go beyond the scope of the adhesion model, because the three types of singularities are very different in regard to the gravitational dynamics. The accumulation of matter in sheets produces density singularities, but the gravitational potential stays finite. In contrast, filaments and nodes are gravitational singularities as well as density singularities, so their formation involves the dissipation of an infinite amount of energy. Therefore, it is not surprising that the analysis of $N$-body cosmological simulations [8] shows that the spectrum of local dimensions $\alpha$ is cut off at $\alpha=1$, which is precisely the local dimension of filaments. Nevertheless, for filaments, the gravitational potential has just a logarithmic singularity, which is milder than the $r^{-1}$ singularity of nodes and, hence, involves less dissipation.

At any rate, gravitational singularities cannot be described in a Newtonian framework and need the Theory of General Relativity. In this theory, the energy dissipated in, for example, the formation of a point singularity as a black hole is finite, namely, it is given by the well-studied black-hole entropy. Plausibly, a good part of the gravitational energy dissipation that takes place below the (cosmic) Kolmogorov scale can be attributed to the formation and growth of super-massive black holes, which occur due to dissipative processes in the dark matter and, preferentially, in the baryonic matter. However, the formation and growth of black holes or other relativistic gravitational singularities is beyond the scope of the adhesion model and even beyond the scope of (state-of-the-art) $N$-body cosmological simulations.

In conclusion, a hydrodynamic closure approach to three-dimensional Burgers turbulence leads to 
Kolmogorov's scaling laws, although in a general form compatible with the presence of strong intermittency. These scaling laws can be applied to the stochastic adhesion model of the cosmic structure, in particular, to the determination of the density two-point correlation function. The result is in partial agreement with the twopoint correlation function obtained from the distribution of galaxies and from $N$-body simulations but suggests that the adhesion model underestimates the contribution of low-dimensional singularities (filaments and nodes) to energy dissipation, whereas $N$-body simulations overestimate it. It is probably necessary to have a better modeling of small-scale dissipative processes, and this modeling may require ingredients from general relativity.

Peebles P. E. J., The Large-scale Structure of the Universe (Princeton University, Princeton, NJ) 1980. VON WeIZsÄcker C. F., Astrophys. J., 114 (1951) 165. Gurbatov S. N. and SAICHEV A. I., Radiophys. Quantum Electron., 27 (1984) 303.

Shandarin S. F. and Zeldovich Ya. B., Rev. Mod. Phys., 61 (1989) 185.
Vergassola M., Dubrulle B., Frisch U. and Noullez A., Astron. Astrophys., 289 (1994) 325.

Sylos Labini F., Montuori M. and Pietronero L., Phys. Rep., 293 (1998) 61.

Jones B. J., Martínez V. J., SaAr E. and Trimble V., Rev. Mod. Phys., 76 (2004) 1211.

GaIte J., Astrophys. J., 658 (2007) 11; JCAP, 03 (2010) 006.

Gaite J., Domínguez A. and PÉRez-Mercader J., Astrophys. J. Lett., $\mathbf{5 2 2}$ (1999) L5.

SAichev A. I. and Woyczynski W. A., SIAM J. Appl. Math., 56 (1996) 1008.

Molchanov S. A., Surgailis D. and Woyczynski W. A., Ann. Appl. Probab., 7 (1997) 200.

Frisch U., Turbulence: The Legacy of A.N. Kolmogorov (Cambridge University Press) 1995.

Medina E., Hwa T., Kardar M. and Zhang Y.-Ch., Phys. Rev. A, 39 (1989) 3053.

Domínguez A., Hochberg D., Martín-García J. M., PÉrez-Mercader J. and Schulman L. S., Astron. Astrophys., 344 (1999) 27.

Barabási A.-L., SzÉPfalusy P. and Vicsek T., Physica A, 178 (1991) 17.

Polyakov A. M., Phys. Rev. E, 52 (1995) 6183.

Hayot F, and Jayaprakash C., Int. J. Mod. Phys. B, $14(2000) 1781$. 